\title{
EL EQUIPAMIENTO Y OTRAS CARACTERISTICAS DE LOS HOGARES EN LAS COMUNIDADES AUTONOMAS
}

$311: 353: 71: 330: 123.6$

por

Ignacio Ballester Ros

SUMARIO: I. INTRODUCCION.-II. LOS HOGARES ESPAÑOLES SEGUN IEL REGIMEN DE TENENCIA DE LA VIVIENDA FAMILIAR.-III. LOS HOGARES ESPAÑOLES SEGUN LA SUPERFICIE UTIL DE LAS VIVIENDAS FAMILIARES.-IV. LOS HOGARES ESPAÑOLES SEGUN LOS SERVICIOS DE HIGIENE DISPONIBLES EN LAS VIVIENDAS FFAMILIARES.-V. LOS HOGARES ESPAÑOLES SEGUN LAS INSTALACIONES EXISTENTES EN LAS VIVIENDAS FAMILIARES: 1. AGUA CORRIENTE. 2. Calefacción. 3. Electricidad, TELÉfono y garaJE.-VI. EL EQUIPAMIENTO DE LOS HOGARES ESPAÑOLES: 1. APARATOS Electrodomésticos. 2. APARATOS DE MÚSICA E IMAGEN. 3. VEHIfCULOS.

\section{INTRODUCCION}

El Instituto Nacional de Estadística acaba de publicar los dos primeros tomos que contienen los resultados de la Encuesta de Presupuestos Familiares 1980-1981. El primer tomo se refiere concretamente a «El gasto y el ingreso de los hogares», y el segundo al 
«Equipamiento y condiciones de las viviendas familiares», con una amplia información relativa al conjunto nacional, a las Comunidades Autónomas, a las Provincias y a los Municipios, agrupados por estratos demográficos.

Como en la propia presentación se expone, la Encuesta de Presupuestos Familiares, cuya recogida de datos primarios se extendió. desde el mes de abril de 1980 al mes de marzo de 1981, responde, en principio, a las mismas necesidades que motivaron la realización de las Encuestas de 1958, 1964-68 y 1973-74, es decir, la puesta al día del conjunto de bienes y servicios que componen la «cesta de la compra» del Indice de Precios al Consumo, sus ponderaciones. y el estrato de referencia, así como el conocimiento de la estructura de los ingresos y gastos de los hogares españoles.

Es evidente que los objetivos de una Encuesta de tal envergadura, dirigida a los hogares, pueden ser ampliados en busca de información sociológica que explique el comportamiento de los hogares. y sus miembros frente a los más diversos temas. $Y$, efectivamente, esta última Encuesta ha sido proyectada para ahondar en diferentes campos de la vivienda, del equipamiento del hogar y de la estructura de los gastos de los hogares en enseñanza, sanidad y alimentación.

Por orden de prioridad, los objetivos de la Encuesta han sido los siguientes:

1. Actualización de la información básica del Indice de Precios de Consumo: bienes y servicios de la "cesta de la compra», sus ponderaciones y el estrato de referencia.

2. Conocimiento de la estructura del presupuesto de los hogares españoles, esto es, de los orígenes de sus ingresos y el montante de los mismos; el destino de estos recursos en la adquisición de bienes y servicios; el ahorro y el endeudamiento. Este conocimiento es sumamente importante, tanto para el análisis social como para la estimación del consumo global de la Contabilidad Nacional.

3. Análisis específicos de determinados campos de preocupación social, como la vivienda, la enseñanza, la sanidad y el equipamiento familiar bajo la perspectiva del gasto.

4. Realización de análisis en el tiempo - series cronológicas, estudios de coyuntura, etc.-, y en el espacio -comparaciones interprovinciales, entre Comunidades Autónomas e internacionales-.

En los años 1968 y 1975 se llevaron a cabo por el Instituto sendas Encuestas específicas sobre Equipamiento de los hogares y Nivel 
cultural de las familias. La parte correspondiente al Equipamiento de los hogares se ha integrado en la reciente Encuesta de Presupuestos Familiares 1980-81, como se ha indicado en los párrafos anteriores.

Gran parte de la información relativa a los hogares y a las viviendas familiares ha sido también objeto de investigación en el Censo de Viviendas de 1981. La coincidencia en el tiempo de ambas investigaciones estadísticas constituye una interesante experiencia, que permitirá apreciar la concordancia de datos y de resultados procedentes de investigaciones realizadas con distinta metodología.

En crónicas anteriores, publicadas en esta Revista, hemos llevado a cabo análisis y comentarios de los resultados obtenidos en el Censo de Edificios de octubre de 1980, en dos fases, y de la primera fase del Censo de Viviendas de marzo de 1981. Por ello, nos ha parecido conveniente dedicar esta crónica a un comentario relativo a los resultados referentes al equipamiento de los hogares y otras características, en especial, de las viviendas familiares, en las que aquéllos están constituidos, información todavía no publicada respecto al Censo de Viviendas de 1981, pero sí abordada en la citada Encuesta de Presupuestos Familiares 1980-81, con lo que cerramos el ciclo informativo referente a los edificios y viviendas en España.

Dada la riqueza informativa, deducida de la Encuesta, en esta materia, nos limitamos al análisis del equipamiento de los hogares y algunas características de las viviendas familiares, referidas exclusivamente, como área territorial considerada, a las Comunidades Autónomas. Los estudiosos pueden profundizar en el estudio referente a otras características no contempladas por nosotros y en el análisis de los resultados provinciales y municipales.

En un momento ulterior abordaremos el comentario de otros resultados, de gran interés, obtenidos en la Encuesta de referencia, con respecto a las materias enumeradas en la reseña de objetivos antes expuesta.

\section{LOS HOGARES ESPAÑOLES SEGUN EL REGIMEN DE TENENCIA DE LA VIVIENDA FAMILIAR}

En el conjunto de España aparecen registrados en la citada Encuesta 10.024.739 hogares, que, según el régimen de tenencia de la propiedad de la vivienda familiar, atendiendo a las distintas formas consideradas, presenta la siguiente distribución: 


\begin{tabular}{|c|c|c|}
\hline & $\begin{array}{c}\text { Cifras } \\
\text { absolutas }\end{array}$ & Porcentajes \\
\hline 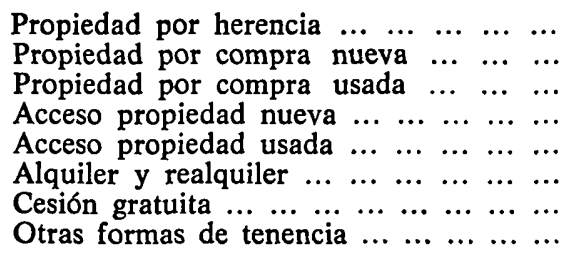 & $\begin{array}{r}1.428 .499 \\
2.504 .304 \\
1.448 .114 \\
1.281 .230 \\
266.576 \\
2.297 .105 \\
686.089 \\
112.822\end{array}$ & $\begin{array}{r}14,2 \\
25,0 \\
14,4 \\
12,8 \\
2,7 \\
22,9 \\
6,8 \\
1,1\end{array}$ \\
\hline
\end{tabular}

Del examen de las cifras anteriores parece deducirse que la adquisición de la propiedad de la vivienda familiar por herencia afecta a la sexta parte de los hogares españoles. La adquisición por compra o en régimen de acceso afecta a más de la mitad —el 54,9 por 100 - de los hogares, el 40 por 100 por compra y la sexta parte por acceso a la propiedad de la vivienda. La adquisición de la propiedad por compra o acceso a vivienda nueva supone también el 40 por 100 , y a la vivienda usada también viene a representar una sexta parte del total. Tan sólo algo más de la quinta parte —el 23 por 100 - de las viviendas familiares principales están ocupadas en régimen de alquiler o realquiler. La cesión gratuita por relaciones familiares o profesionales no rebasa el 7 por 100 , y las demás formas de tenencia apenas rebasan el 1 por 100 .

Si tenemos en cuenta la distribución de los hogares, según el régimen de tenencia de la vivienda familiar en las Encuestas antes. citadas y en la actual, resulta la siguiente distribución:

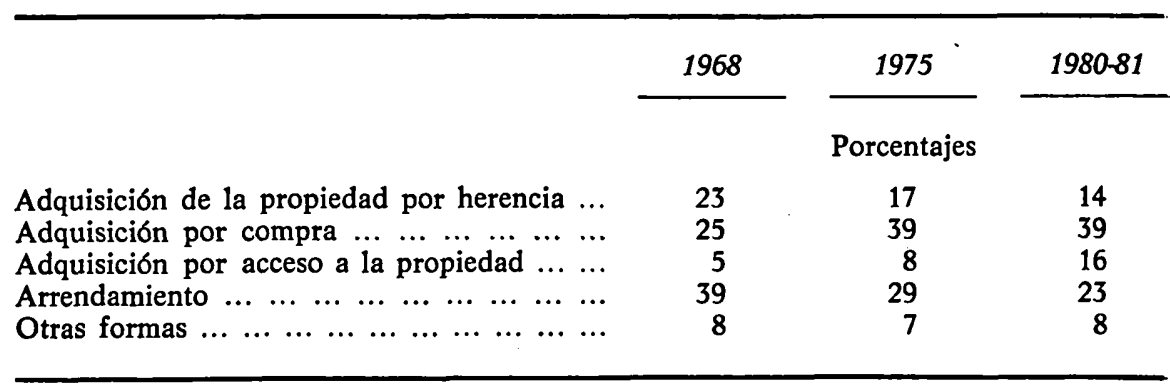

Mientras se apunta una tendencia decreciente de la herencia como forma de adquisición de la propiedad de las viviendas fami- 
liares principales, y asimismo, del régimen de arrendamiento, se advierte una tendencia creciente de la compra de viviendas nuevas o usadas, que se acentuó en el período 1968-1975 y se ha mantenido estacionaria en el período 1975-1981, y de una tendencia al acceso a la propiedad, que si parte de una base más débil en 1968, se acentúa acusadamente, hasta duplicarse, en el período 1975-1981. Las restantes formas de tenencia se mantienen estacionarias, con valores muy semejantes en los tres momentos considerados.

La distribución de los hogares por Comunidades Autónomas es como sigue:

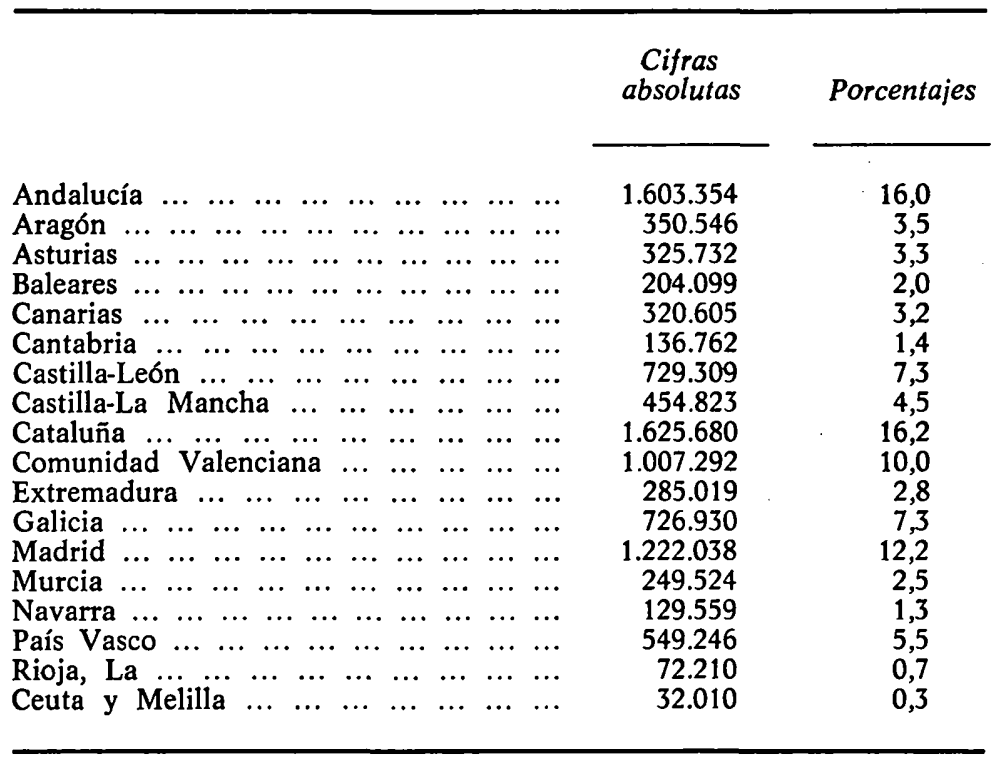

En esta distribución destacan cuatro Comunidades que superan el millón de hogares: Cataluña y Andalucía, con más de millón y medio cada una; Madrid, con un millón y cuarto, y Valencia, que supera ligeramente el millón. En estas cuatro Comunidades están constituidos más de la mitad de los hogares españoles.

A segundo nivel figuran Castilla-León y Galicia, con algo más de 700.000 hogares; el País Vasco, que supera el medio millón, y Castilla-La Mancha, con más de 450.000; Aragón, Asturias y Canarias, que rebasan los 300.000; Extremadura, que se acerca a $300.000, \mathrm{y}$ Murcia, a los 250.000; Baleares, que apenas supera los 200.000; 
Cantabria y Navarra, por encima de los 100.000, y, finalmente, La Rioja, que no alcanza, con mucho, los 100.000 .

La distribución de los hogares según el régimen de tenencia de la vivienda familiar principal, por Comunidades Autónomas, es como sigue:

\begin{tabular}{|c|c|c|c|c|c|}
\hline & Herencia & Compra & $\begin{array}{l}\text { Acceso a la } \\
\text { propiedad }\end{array}$ & $\begin{array}{l}\text { Arrenda- } \\
\text { miento }\end{array}$ & $\begin{array}{c}\text { Otras } \\
\text { formas }\end{array}$ \\
\hline & \multicolumn{5}{|c|}{ Porcentajes } \\
\hline $\begin{array}{llll}\text { Andalucía } & \ldots & \ldots & \ldots\end{array}$ & 12,8 & 40,8 & 17,5 & 19,1 & 9,8 \\
\hline 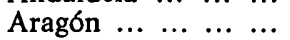 & 19,9 & 42,5 & 9,5 & 18,9 & 9,2 \\
\hline 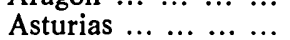 & 18,8 & 35,3 & 7,9 & 30,2 & 7,8 \\
\hline Baleares $\ldots \ldots \ldots \ldots$ & 16,5 & 38,3 & 6,5 & 30,3 & 8,4 \\
\hline $\begin{array}{lllll}\text { Canarias... } & \ldots & \ldots & \ldots\end{array}$ & 15,3 & 43,8 & 10,8 & 17,3 & 12,8 \\
\hline $\begin{array}{cccc}\text { Cantabria } & \ldots & \ldots & \ldots\end{array}$ & 11,5 & 48,9 & 8,3 & 19,8 & 11,5 \\
\hline Castilla-León $\quad . . . \quad \ldots$ & 19,6 & 42,9 & 9,5 & 18,8 & 9,2 \\
\hline Castilla-La Mancha.. & 22,7 & 47,5 & 7,0 & 13,0 & 9,8 \\
\hline Cataluña... ......... & 9,4 & 28,6 & 19,4 & 37,2 & 5,4 \\
\hline Comunidad Valen- & & & & & \\
\hline $\begin{array}{ccccc}\text { ciana } & \ldots & \ldots & \ldots & \ldots\end{array}$ & 14,8 & 39,7 & 20,5 & 16,9 & 8,1 \\
\hline $\begin{array}{lll}\text { Extremadura } & \ldots & \ldots\end{array}$ & 23,4 & 40,7 & 8,3 & 15,7 & 11,9 \\
\hline $\begin{array}{lllll}\text { Galicia } & \ldots & \ldots & \ldots & \ldots\end{array}$ & 30,6 & 36,3 & 4,0 & 18,7 & 10,4 \\
\hline Madrid $\ldots \ldots \ldots \ldots$ & 2,5 & 38,5 & 25,8 & 28,3 & 4,9 \\
\hline $\begin{array}{ccccc}\text { Murcia } & \ldots & \ldots & \ldots & \ldots\end{array}$ & 22,8 & 49,8 & 7,3 & 11,8 & 8,3 \\
\hline 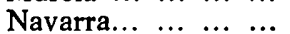 & 14,3 & 42,7 & 22,8 & 14,2 & 6,0 \\
\hline País Vasco $\ldots \ldots \ldots$ & 7,1 & 49,4 & 19,2 & 19,3 & 5,0 \\
\hline Rioja, La $\ldots \begin{array}{lll} & \ldots & \ldots\end{array}$ & 12,4 & 59,4 & 3,8 & 18,3 & 6,1 \\
\hline Ceuta y Melilla ... & 7,5 & 25,4 & 4,2 & 51,8 & 11,1 \\
\hline
\end{tabular}

La tenencia, por los hogares, de la vivienda nueva o usada, por compra, está muy generalizada en todo el país. Doce Comunidades Autónomas presentan, en este aspecto, porcentajes superiores al promedio nacional y configuran una gran zona, sin solución de continuidad, que comprende Cantabria, el País Vasco, La Rioja, Navarra y Aragón, ambas Castillas y Extremadura, Valencia y Murcia, Andalucía y Canarias. Las demás Comunidades Autónomas -Asturias y Galicia, Madrid, Baleares y Cataluña- presentan porcentajes no muy alejados del promedio nacional, con la excepción de Cataluña y de Ceuta y Melilla. Ello demuestra que el fenómeno se da prácticamente en toda la Nación.

El acceso a la propiedad de la vivienda, nueva o usada, no presenta la misma uniformidad en su desarrollo y ofrece, por el contrario, una marcada discrepancia de unas a otras Comunidades 
Autónomas. Tan sólo en Madrid, País Vasco y Navarra, Valencia, Cataluña y Andalucía sobresalen, sobre el conjunto nacional, por esta forma de tenencia de la vivienda familiar. En las restantes Comunidades tienen claramente una menor importancia.

$\mathrm{El}$ arrendamiento es una forma habitual, utilizada en todas las Comunidades Autónomas, que afecta, a lo menos, entre la sexta y la quinta parte de sus hogares, si bien destaca sobremanera en Cataluña, Baleares, Asturias y Madrid, y muy singularmente en Ceuta y Melilla. Castilla-La Mancha y Murcia presentan los valores mínimos de esta incidencia.

También la herencia está muy extendida territorialmente y afecta, con sus valores superiores al promedio nacional, a once Comunidades Autónomas: Galicia y Asturias, ambas Castillas y Extremadura, Aragón y Navarra, Valencia y Murcia, y las islas Baleares y Canarias. Por el contrario, Madrid, el País Vasco y Cataluña presentan tasas muy bajas.

La cesión gratuita y otras formas de tenencia de la vivienda se da principalmente en Canarias, Galicia, Cantabria y Extremadura, así como en Ceuta y Melilla, y a segundo nivel, en ambas Castillas, Aragón, Andalucía, Baleares, Valencia y Murcia.

\section{LOS HOGARES ESPAÑOLES SEGUN LA SUPERFICIE UTIL DE LAS VIVIENDAS FAMILIARES}

La distribución de las viviendas familiares según su superficie útil es como sigue:

\begin{tabular}{|c|c|c|c|c|}
\hline $\begin{array}{c}\text { De hasta } \\
50 \mathrm{~m}^{2}\end{array}$ & $\begin{array}{c}\text { De } 51 \\
a 80\end{array}$ & $\begin{array}{l}\text { De } 81 \\
a \quad 100\end{array}$ & $\begin{array}{cc}\text { De } 101 \\
\text { a } 130\end{array}$ & $\begin{array}{l}\text { De } 131 \\
\text { y más }\end{array}$ \\
\hline \multicolumn{5}{|c|}{ Porcentajes } \\
\hline 11,0 & 46,0 & 25,4 & 10,6 & 7,0 \\
\hline 14,4 & 41,8 & 25,6 & 10,1 & 8,1 \\
\hline 9,4 & 48,9 & 21,4 & 11,3 & 9,0 \\
\hline 15,6 & 56,5 & 18,6 & 6,3 & 3,0 \\
\hline 3,8 & 30,9 & 35,8 & 17,5 & 12,0 \\
\hline 13,2 & 37,6 & 25,3 & 13,8 & 10,1 \\
\hline 6,2 & 43,3 & 33,3 & 10,2 & 7,0 \\
\hline 8,2 & 43,7 & 31,2 & 10,5 & 6,4 \\
\hline 12,6 & 40,5 & 25,6 & 12,2 & 9,1 \\
\hline 6,2 & 59,3 & 21,6 & 7,8 & 5,1 \\
\hline
\end{tabular}




\begin{tabular}{|c|c|c|c|c|c|}
\hline & $\begin{array}{c}\text { De hasta } \\
50 \mathrm{~m}^{2}\end{array}$ & $\begin{array}{c}\text { De } 51 \\
\text { a } 80\end{array}$ & $\begin{array}{l}\text { De } 81 \\
a 100\end{array}$ & $\begin{array}{cc}\text { De } & 101 \\
\text { a } & 130\end{array}$ & $\begin{array}{l}\text { De } 131 \\
\text { y más }\end{array}$ \\
\hline & \multicolumn{5}{|c|}{ Porcentajes } \\
\hline 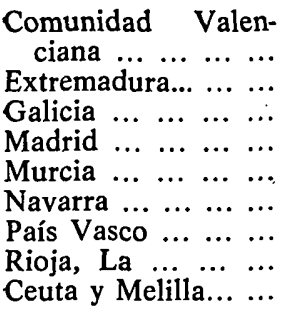 & $\begin{array}{r}4,3 \\
10,6 \\
16,4 \\
21,0 \\
3,8 \\
5,1 \\
5,1 \\
9,3 \\
26,4\end{array}$ & $\begin{array}{l}39,7 \\
40,0 \\
42,5 \\
49,0 \\
21,0 \\
34,6 \\
57,5 \\
47,4 \\
50,8\end{array}$ & $\begin{array}{l}33,6 \\
28,9 \\
25,2 \\
18,4 \\
31,5 \\
35,2 \\
22,6 \\
31,1 \\
17,5\end{array}$ & $\begin{array}{r}16,2 \\
14,7 \\
8,9 \\
6,6 \\
25,3 \\
13,5 \\
7,9 \\
8,1 \\
3,4\end{array}$ & $\begin{array}{r}6,2 \\
5,8 \\
7,0 \\
5,0 \\
18,4 \\
11,6 \\
6,9 \\
4,1 \\
1,9\end{array}$ \\
\hline
\end{tabular}

En el conjunto nacional predominan las viviendas familiares de superficie comprendida entre los 51 y 100 metros cuadrados, que representan el 71,4 por 100 del total de viviendas. Casi la mitad tienen una superficie de 51 a 80 metros cuadrados, y la cuarta parte tienen una superficie entre 81 y 100 metros cuadrados. Las viviendas con superficie inferior a 50 metros cuadrados y las con superficie entre 101 y 130 metros cuadrados suponen un 11 por 100 cada uno de los dos grupos considerados. Las viviendas superiores a los 130 metros cuadrados de superficie son el 7 por 100 del total. La superficie media por miembro del hogar es de 22,8 metros cuadrados, y el número de miembros del hogar por habitación es de 0,74.

También predominan en todas las Comunidades Autónomas las viviendas familiares de superficie comprendida entre los 51 y 100 metros cuadrados, que en Cataluña y el País Vasco representan las cuatro quintas partes del total respectivo; superan el promedio nacional en Asturias, Cantabria, Castilla-León, La Rioja y Valencia; representan entre el 60 y el 70 por 100 en las demás Comunidades Autónomas, con la excepción de Murcia, que ligeramente supera la mitad.

Más de la mitad de las viviendas de Cataluña, el País Vasco, Asturias y Ceuta y Melilla tienen una superficie entre 51 y 80 metros cuadrados, y superan el 40 por 100 las demás Comunidades Autónomas, con la excepción de Navarra, Baleares y Canarias -entre el 30 y el 40 por 100-, y Murcia, sólo con el 21. Valencia tiene prácticamente el 40 por 100 .

Las viviendas de superficie comprendida entre los 51 y 80 metros 
cuadrados superan el promedio nacional en Cantabria, Navarra y La Rioja, ambas Castillas, Extremadura, Valencia y Murcia, y Andalucía. Se aproximan a dicho promedio Galicia y Canarias. Las demás Comunidades superan, en unos casos, la sexta parte, y en otros, la quinta parte del total.

Las viviendas familiares de superficie entre 101 y 130 metros cuadrados destacan en Murcia, en la que representan la cuarta parte del total de viviendas, y le siguen en orden de importancia Valencia y Baleares, Extremadura, Canarias y Navarra, Castilla-La Mancha y Aragón. Se acercan al promedio nacional Castilla-León, Cantabria y Andalucía. Las demás Comunidades presentan tasas inferiores.

Las viviendas con superficie igual o superiores a los 131 metros cuadrados sobresalen numéricamente en Murcia, Navarra, Baleares y Canarias, siguiéndoles a segundo nivel, Cantabria, Galicia, Aragón, Castilla-La Mancha y Andalucía.

Con viviendas de superficie hasta 50 metros cuadrados destacan Ceuta, Melilla y Madrid, con más de la quinta parte del total de viviendas, seguidas de Galicia, Asturias, Castilla-La Mancha, Andalucía y Canarias.

La superficie útil por miembro del hogar oscila entre 18,7 de Ceuta y Melilla, 32 de Baleares y 28,2 de Murcia. Las restantes Comunidades presentan valores entre el 21,2 y el 26,9 , con la excepción de Madrid, que no llega al 20.

El número de miembros del hogar por habitación es muy similar entre todas las Comunidades Autónomas y oscila entre 0,60 para Baleares, 0,82 para Ceuta y Melilla y 0,93 para Canarias. Los valores medios predominantes van de 0,67 a 0,78 .

\section{LOS HOGARES ESPAÑOLES SEGUN LOS SERVICIOS DE HIGIENE DISPONIBLES EN LAS VIVIENDAS FAMILIARES}

La distribución de los hogares según los servicios de higiene de que disponen, por Autonomías, es la siguiente: 


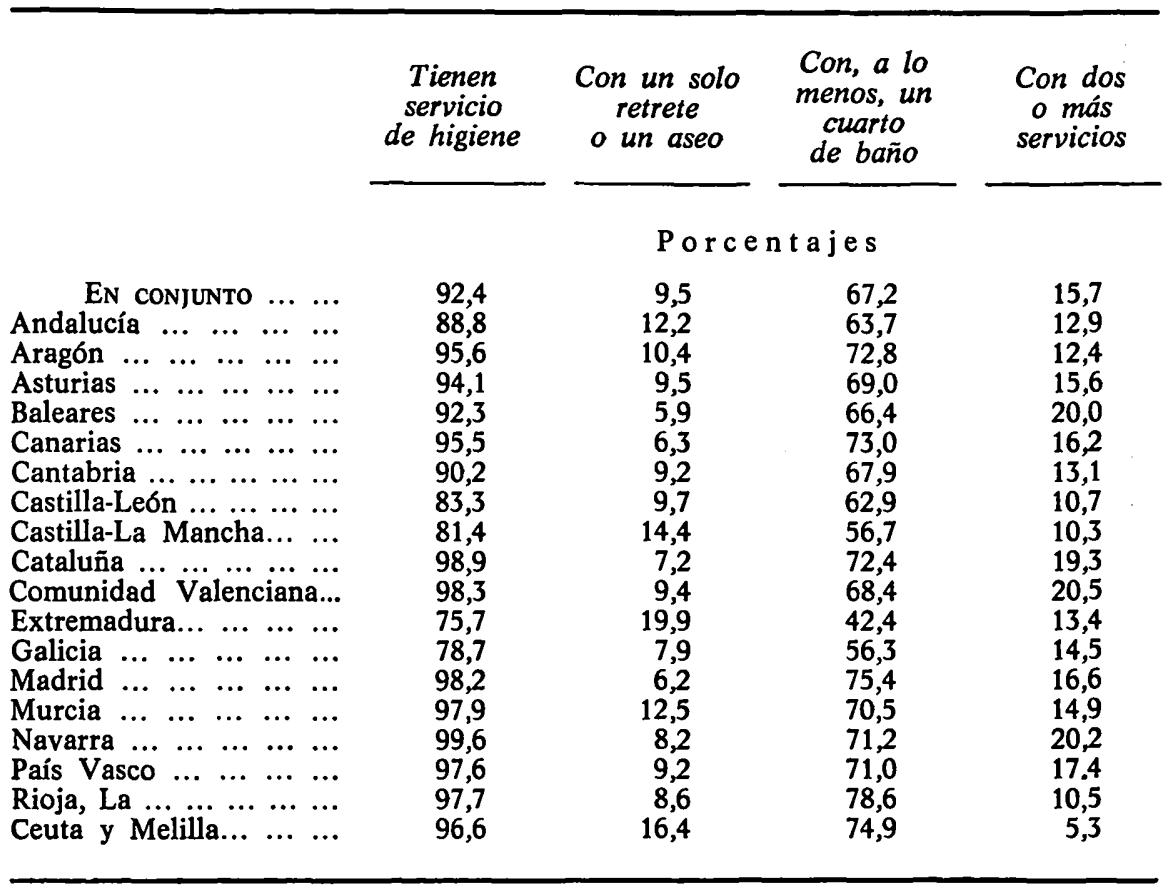

En el conjunto nacional disponen de servicio de higiene en los hogares el 92,4 por 100 . Más de los dos tercios de los hogares disponen de, a lo menos, un cuarto de baño; una sexta parte disponen de dos, tres o más servicios, y tan sólo una décima parte tienen solamente un retrete o un aseo. Quedan todavía un 7,6 por 100 de los hogares sin servicios de higiene o con servicios compartidos.

La evolución registrada en la disponibilidad de los servicios de higiene en los hogares se deduce de las siguientes cifras correspondientes a las dos Encuestas anteriores, ya citadas, y a la más reciente:

\begin{tabular}{|c|c|c|c|c|}
\hline & $\begin{array}{l}\text { Sin servicio } \\
\text { o compartido }\end{array}$ & $\begin{array}{l}\text { Solo aseo } \\
\text { o retrete }\end{array}$ & $\begin{array}{l}\text { Con solo } \\
\text { cuarto } \\
\text { de baño }\end{array}$ & $\begin{array}{l}\text { Con dos } \\
\text { o más } \\
\text { servicios }\end{array}$ \\
\hline & \multicolumn{4}{|c|}{ Porcentajes } \\
\hline $\begin{array}{lllll}\text { Encuesta de } & 1968 & \ldots & \ldots \\
\text { Encuesta de } & 1975 & \ldots & \ldots \\
\text { Encuesta de } & 1981 & \ldots & \ldots\end{array}$ & $\begin{array}{r}35 \\
17 \\
8\end{array}$ & $\begin{array}{r}44 \\
25 \\
9\end{array}$ & $\begin{array}{l}20 \\
52 \\
67\end{array}$ & $\begin{array}{r}1 \\
6 \\
16\end{array}$ \\
\hline
\end{tabular}


De las cifras anteriores, se deduce claramente la extraordinaria mejora en la disponibilidad de los servicios de higiene en los hogares españoles registrada en trece años.

El servicio de cuarto de baño se ha generalizado en los hogares de todas las Comunidades Autónomas; en doce de ellas esta disponibilidad supera el promedio nacional. Baleares se acerca a este promedio. Más del 60 por 100 de los hogares de Castilla-León y más de la mitad de los de Castilla-La Mancha y Galicia disponen también de este servicio. Ocupa el último lugar Extremadura, con el 42 por 100 de los hogares disponiendo de este servicio.

A lo menos, el 10 por 100 de los hogares de todas las Comunidades disponen de dos, tres o más servicios. En siete de ellas rebasan el promedio nacional, y en algunas de ellas superan el 20 por 100 los hogares que disponen de este número de servicios. Estas Comunidades son, por orden de importancia, Valencia, Navarra y Baleares, seguidas de Cataluña, País Vasco, Madrid y Canarias.

Con un solo retrete o aseo se sitúan, con valores superiores al promedio nacional: Asturias, ambas Castillas y Extremadura, Aragón, Murcia, Andalucía y Ceuta y Melilla.

La carencia de estos servicios se hace más notoria en Extremadura y Galicia, ambas Castillas, Andalucía, Cantabria y Baleares.

\section{LOS HOGARES ESPAÑOLES SEGUN LAS INSTALACIONES EXISTENTES EN LAS VIVIENDAS FAMILIARES}

Las instalaciones que básicamente se analizan son las de: agua; calefacción; electricidad, teléfono y garaje.

\section{Agua CORRIENTE}

La distribución de los hogares en orden a las instalaciones para el suministro de agua es la siguiente: 


\begin{tabular}{|c|c|c|c|c|}
\hline & $\begin{array}{l}\text { Sin agua } \\
\text { corriente }\end{array}$ & $\begin{array}{l}\text { Con agua } \\
\text { corriente } \\
\text { fría }\end{array}$ & $\begin{array}{l}\text { Con agua } \\
\text { fría y } \\
\text { calentador } \\
\text { individual }\end{array}$ & $\begin{array}{c}\text { Con agua } \\
\text { fria y } \\
\text { caliente } \\
\text { central }\end{array}$ \\
\hline & \multicolumn{4}{|c|}{ Porcentajes } \\
\hline EN CONJUNTO $\ldots \ldots$ & 3,8 & 19,5 & 71,4 & 5,3 \\
\hline $\begin{array}{lllll}\text { Andalucía } & \ldots & \ldots & \ldots & \ldots\end{array}$ & 6,1 & 25,2 & 66,8 & 1,9 \\
\hline $\begin{array}{lllllll}\text { Aragón } & \ldots & \ldots & \ldots & \ldots & \ldots\end{array}$ & 1,8 & 17,7 & 67,9 & 12,6 \\
\hline $\begin{array}{llllll}\text { Asturias } & \ldots & \ldots & \ldots & \ldots & \ldots\end{array}$ & 2,3 & 17,8 & 71,7 & 8,2 \\
\hline $\begin{array}{llllll}\text { Baleares } & \ldots & \ldots & \ldots & \ldots & \ldots \\
\end{array}$ & 9,7 & 23,8 & 59,6 & 6,9 \\
\hline $\begin{array}{lllll}\text { Canarias } & \ldots & \ldots & \ldots & \ldots\end{array}$ & 4,2 & 39,9 & 55,3 & 0,6 \\
\hline $\begin{array}{lllll}\text { Cantabria } & \ldots & \ldots & \ldots & \ldots\end{array}$ & 3,3 & 12,2 & 78,7 & 5,8 \\
\hline Castilla-León ... ... ........ & 6,1 & 22,9 & 64,4 & 6,6 \\
\hline Castilla-La Mancha... ... & 9,4 & 24,0 & 63,1 & 3,5 \\
\hline $\begin{array}{llllll}\text { Cataluña } & \ldots & \ldots & \ldots & \ldots & \ldots\end{array}$ & 1,5 & 15,7 & 79,8 & 3,0 \\
\hline Comunidad Valenciana... & 1,2 & 19,0 & 78,2 & 1,6 \\
\hline Extremadura $\ldots \ldots \ldots$ & 11,4 & 41,9 & 45,1 & 1,6 \\
\hline 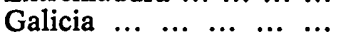 & 8,9 & 31,2 & 55,6 & 4,3 \\
\hline $\begin{array}{llllll}\text { Madrid } & \ldots & \ldots & \ldots & \ldots & \ldots\end{array}$ & 0,6 & 5,9 & 79,9 & 13,6 \\
\hline $\begin{array}{llllll}\text { Murcia } & \ldots & \ldots & \ldots & \ldots & \ldots\end{array}$ & 1,1 & 11,7 & 86,6 & 0,6 \\
\hline 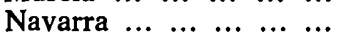 & 0,2 & 8,7 & 73,8 & 17,3 \\
\hline $\begin{array}{lllll}\text { País Vasco } & \ldots & \ldots & \ldots & \ldots\end{array}$ & 0,6 & 6,5 & 84,4 & 8,5 \\
\hline Rioja, La ... ........... & 0,4 & 13,2 & 76,0 & 10,4 \\
\hline Ceuta y Melilla ... ... ... & 3,0 & 20,0 & 75,5 & 1,5 \\
\hline
\end{tabular}

En el conjunto nacional, el 96,2 por 100 de los hogares españoles disponen de agua corriente, el 77 por 100 de agua caliente -central o individual-y sólo el 4 por 100 no disponen de ella.

Según las tres Encuestas a las que nos referimos en esta crónica, la evolución en la disponibilidad de agua corriente, fría y caliente, es como sigue:

$\underbrace{\begin{array}{c}\text { Agua } \\ \text { corriente fria }\end{array}}_{\begin{array}{c}\text { Sin agua } \\ \text { corriente }\end{array}} \begin{gathered}\begin{array}{c}\text { Agua fría } \\ y \text { caliente }\end{array} \\ \text { Porcentajes }\end{gathered}$

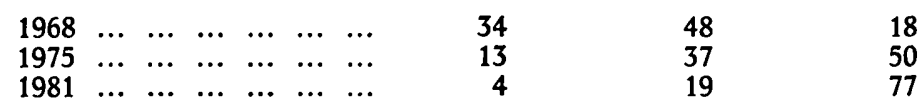

Claramente se deduce de las cifras anteriores la extraordinaria mejora registrada en los hogares españoles en orden a la disponibilidad de agua corriente, fría y caliente. 
Más del 90 por 100 de los hogares de Madrid, País Vasco y Navarra disponen de agua fría y caliente central o por calentador individual. Lo propio ocurre con más del 80 por 100 de los hogares de Cataluña, Murcia, La Rioja, Cantabria y Aragón, y entre el 71 y el 80 por 100 de los de Asturias, Valencia y Ceuta y Melilla. Los valores mínimos, en este orden, corresponden a Extremadura y Galicia, Baleares y Canarias.

Destacan por la disponibilidad de agua caliente central: Navarra, Madrid, Aragón y La Rioja, en primer término, y en segundo término, Asturias, Cantabria, el País Vasco, Castilla-León y Baleares. Las demás Comunidades presentan valores mucho más modestos.

Disponen de agua corriente fría, con valores superiores al promedio nacional, los hogares de Galicia, ambas Castillas, Extremadura, Andalucía, Baleares y Canarias, Ceuta y Melilla.

Finalmente, no disponen de agua corriente en los hogares el 4 por 100 del total, si bien esta deficiencia se manifiesta de forma más patente en Galicia, Extremadura, ambas Castillas, Andalucía y Baleares y Canarias.

\section{Calefacción}

La distribución de los hogares en cuanto a la instalación de calefacción es la siguiente:

Individual

$\frac{\begin{array}{c}\text { Tienen } \\ \text { calefaccion }\end{array}}{\text { Porcentajes }} \frac{\begin{array}{c}\text { Con aparatos } \\ \text { móviles }\end{array}}{\begin{array}{c}\text { Con aparatos } \\ \text { fijos }\end{array}}$ Central

\begin{tabular}{|c|c|c|c|c|}
\hline EN CONJUNTO $\ldots \ldots$ & 38,3 & 21,1 & 8,8 & 8,5 \\
\hline $\begin{array}{lllll}\text { Andalucía } & \ldots & \ldots & \ldots & \ldots\end{array}$ & 18,6 & 16,1 & 0,9 & 1,6 \\
\hline $\begin{array}{llllll}\text { Araggon } & \ldots & \ldots & \ldots & \ldots & \ldots\end{array}$ & 65,2 & 29,9 & 14,9 & 20,4 \\
\hline $\begin{array}{llllll}\text { Asturias } & \ldots & \ldots & \ldots & \ldots & \ldots\end{array}$ & 36,1 & 11,4 & 14,7 & 10,0 \\
\hline $\begin{array}{lllllll}\text { Baleares } & \ldots & \ldots & \ldots & \ldots & \ldots\end{array}$ & 34,9 & 29,5 & 3,2 & 2,2 \\
\hline $\begin{array}{lllllll}\text { Canarias } & \ldots & \ldots & \ldots & \ldots & \ldots\end{array}$ & 7,4 & 7,3 & - & 0,1 \\
\hline $\begin{array}{ccccc}\text { Cantabria } & \ldots & \ldots & \ldots & \ldots\end{array}$ & 36,1 & 18,7 & 9,7 & 7,7 \\
\hline Castilla-León $\ldots \ldots \ldots \ldots$ & 44,4 & 15,9 & 18,1 & 10,4 \\
\hline Castilla-La Mancha ... ... & 30,8 & 16,5 & 5,2 & 9,1 \\
\hline $\begin{array}{cccccc}\text { Cataluña } & \ldots & \ldots & \ldots & \ldots & \ldots\end{array}$ & 33,9 & 16,6 & 12,3 & 5,0 \\
\hline
\end{tabular}




\begin{tabular}{|c|c|c|c|c|}
\hline \multirow{3}{*}{. } & & \multicolumn{2}{|c|}{ Individual } & \multirow{2}{*}{ Central } \\
\hline & $\begin{array}{c}\text { Tienen } \\
\text { calefacción }\end{array}$ & $\begin{array}{c}\text { Con aparatos } \\
\text { móviles }\end{array}$ & $\begin{array}{c}\text { Con aparatos } \\
\text { fijos }\end{array}$ & \\
\hline & \multicolumn{4}{|c|}{ Porcentajes } \\
\hline Comunidad Valenciana... & 39,4 & 34,2 & 3,7 & 1,5 \\
\hline Extremadura $\ldots \ldots \ldots \ldots$ & 10,3 & 7,0 & 0,8 & 2,5 \\
\hline $\begin{array}{cccccc}\text { Galicia } & \ldots & \ldots & \ldots & \ldots & \ldots\end{array}$ & 15,7 & 5,0 & 5,8 & 4,9 \\
\hline $\begin{array}{llllll}\text { Madrid } \ldots & \ldots & \ldots & \ldots & \ldots\end{array}$ & 67,1 & 24,6 & 15,0 & 27,5 \\
\hline $\begin{array}{llllll}\text { Murcia } & \ldots & \ldots & \ldots & \ldots & \ldots\end{array}$ & 49,7 & 45,7 & 2,8 & 1,2 \\
\hline 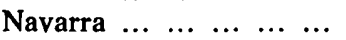 & 58,9 & 5,9 & 23,2 & 29,8 \\
\hline País Vasco $\ldots . . . . . .$. & 79,3 & 55,2 & 13,6 & 10,5 \\
\hline Rioja, La $\ldots \begin{array}{lll} & \ldots & \ldots\end{array}$ & 46,5 & 16,1 & 15,1 & 15,3 \\
\hline Ceuta y Melilla ......... & 28,0 & 22,3 & 5,3 & 0,4 \\
\hline
\end{tabular}

En el conjunto nacional, disponen de calefacción la tercera parte de los hogares españoles, el 30 por 100 de carácter individual y tan sólo el 9 por 100 con calefacción central.

La calefacción central se da preferentemente en hogares de Asturias, País Vasco, La Rioja, Navarra, Aragón, ambas Castillas y Madrid, afectando, como mínimo, al 10 por 100 de los hogares, y como máximo, al 30 por 100 .

La calefacción individual con aparatos fijos, se da también con preferencia en las mismas Comunidades enumeradas por la disponibilidad de calefacción central y, además, en Cataluña y Cantabria. Con aparatos móviles, la calefacción está más extendida. Con la salvedad de Canarias, Extremadura, Galicia y el País Vasco, las demás Comunidades disponen de este tipo de calefacción entre el 10 y el 20 por 100 de sus hogares, nivel que se rebasa en Madrid, Aragón, Baleares y Ceuta y Melilla, acercándose al 30 por 100; Valencia, con más del 30 por 100, y Murcia y el País Vasco, con el 45 y el 55 por 100 de los hogares, respectivamente.

En resumen, son ocho las Comunidades Autónomas que destacan por el número de hogares que disponen de calefacción en cualquiera de sus formas, figurando, en primer lugar, el País Vasco, Madrid y Aragón, con más de los dos tercios de sus hogares disponiendo de estas instalaciones, seguidas de Navarra, con más de la mitad; Murcia, La Rioja y Castilla-León, con más del 40 por 100, y Valencia, con cerca del 40 por 100 de sus hogares. 


\section{Electricidad, TELÉFono y garaJe}

La distribución de los hogares en orden a la instalación de energía eléctrica, teléfono y garaje es como sigue:

\begin{tabular}{|c|c|c|c|c|}
\hline & \multicolumn{4}{|c|}{$H O G A R E S$} \\
\hline & $\begin{array}{l}\text { Que no tienen } \\
\text { electricidad }\end{array}$ & & $\begin{array}{c}\text { Que tienen } \\
\text { teléfono }\end{array}$ & $\begin{array}{l}\text { Que tienen } \\
\text { garaje }\end{array}$ \\
\hline & \multicolumn{4}{|c|}{ Porcentajes } \\
\hline$: \quad$ EN CONJUNTO $\ldots \ldots \ldots \ldots$ & 0,9 & & 50,7 & 16,3 \\
\hline $\begin{array}{lllllll}\text { Andalucía } & \ldots & \ldots & \ldots & \ldots & \ldots & \ldots\end{array}$ & 2,0 & & 31,4 & 9,8 \\
\hline 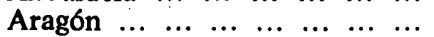 & 0,2 & .. & 61,4 & 24,6 \\
\hline $\begin{array}{llllllll}\text { Asturias } & \ldots & \ldots & \ldots & \ldots & \ldots & \ldots & \ldots\end{array}$ & 1,2 & & 48,4 & 22,2 \\
\hline $\begin{array}{cccccccc}\text { Baleares } & \ldots & \ldots & \ldots & \ldots & \ldots & \ldots & \ldots\end{array}$ & 0,8 & & 48,2 & 24,5 \\
\hline $\begin{array}{cccccccc}\text { Canarias } & \ldots & \ldots & \ldots & \ldots & \ldots & \ldots & \ldots\end{array}$ & 3,8 & & 44,6 & 12,2 \\
\hline $\begin{array}{lllllll}\text { Cantabria } & \ldots & \ldots & \ldots & \ldots & \ldots & \ldots\end{array}$ & 0,5 & & 46,7 & 23,4 \\
\hline Castilla-León $\ldots \ldots \ldots \ldots \ldots$ & 0,4 & & 40,0 & 19,8 \\
\hline Castilla-La Mancha $\ldots \ldots \ldots \ldots$ & 2,1 & & 31,1 & 13,1 \\
\hline $\begin{array}{llllllll}\text { Cataluña } & \ldots & \ldots & \ldots & \ldots & \ldots & \ldots\end{array}$ & 0,2 & & 66,3 & 24,9 \\
\hline Comunidad Valenciana... ... ... & 0,3 & & 48,4 & 15,7 \\
\hline 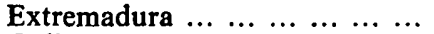 & 1,0 & & 28,5 & 6,5 \\
\hline $\begin{array}{cccccccc}\text { Galicia } & \ldots & \ldots & \ldots & \ldots & \ldots & \ldots & \ldots\end{array}$ & 1,5 & & 30,3 & 14,6 \\
\hline $\begin{array}{llllllll}\text { Madrid } & \ldots & \ldots & \ldots & \ldots & \ldots & \ldots & \ldots\end{array}$ & 0,3 & & 82,1 & 10,3 \\
\hline $\begin{array}{llllllll}\text { Murcia } & \ldots & \ldots & \ldots & \ldots & \ldots & \ldots & \ldots\end{array}$ & 0,7 & & 33,3 & 16,5 \\
\hline $\begin{array}{cccccccc}\text { Navarra } & \ldots & \ldots & \ldots & \ldots & \ldots & \ldots & \ldots\end{array}$ & - & - & 58,8 & 23,2 \\
\hline 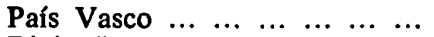 & 0,2 & & 72,2 & 18,6 \\
\hline 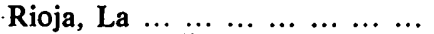 & 0,3 & & 45,0 & 11,0 \\
\hline Ceuta y Melilla $\ldots \ldots \ldots \ldots \ldots$ & 1,5 & & 34,5 & 3,4 \\
\hline
\end{tabular}

Prácticamente, todos los hogares españoles disponen de instalación eléctrica. Tan sólo un 1 por 100 no dispone de ella todavía. Canarias, Castilla-La Mancha, Andalucía, Galicia, Asturias, Extremadura y Ceuta y Melilla son las Comunidades más afectadas por esta carencia.

La mitad de los hogares españoles disponen de teléfono. Figuran a la cabeza: Madrid, cuyas cuatro quintas partes de sus hogares disponen de este servicio; el País Vasco y Cataluña, con más de los dos tercios de hogares; Aragón y Navarra, con cerca de los dos tercios de hogares. En las demás Comunidades disponen de teléfono, como mínimo, una tercera parte de sus hogares, llegando en algunas de ellas a casi la mitad. Los valores mínimos corresponden a Extremadura y Galicia. 
La sexta parte de los hogares españoles disponen de garaje, bien en el propio edificio en que se ubica la vivienda familiar o fuera de él. Con las excepciones de Extremadura y Andalucía, las demás Comunidades Autónomas disponen de este servicio entre el 10 por 100 y algo más de la cuarta parte de sus hogares. Cataluña, Baleares y Asturias, Aragón, Cantabria y Navarra ocupan los primeros lugares.

\section{EL EQUIPAMIENTO DE LOS HOGARES ESPAÑOLES}

La información sobre esta materia alcanza a los aparatos electrodomésticos, los aparatos de música e imagen y los vehículos.

\section{Aparatos electrodomésticos}

Los hogares españoles están equipados con los siguientes aparatos electrodomésticos:

\begin{tabular}{|c|c|c|c|c|}
\hline & & & & \\
\hline EN CONIUNTO $\ldots \ldots$ & 91,1 & 64,3 & 6,3 & 17,7 \\
\hline $\begin{array}{lllll}\text { Andalucía } & \ldots & \ldots & \ldots & \ldots\end{array}$ & 89,3 & 56,4 & 3,7 & 6,4 \\
\hline $\begin{array}{llllll}\text { Aragón } & \ldots & \ldots & \ldots & \ldots & \ldots\end{array}$ & 93,9 & 67,4 & 7,3 & 13,5 \\
\hline $\begin{array}{lllll} & \ldots & \ldots & \ldots & \ldots\end{array}$ & 86.6 & 74,6 & 5,3 & 24,3 \\
\hline $\begin{array}{cccccc}\text { Baleares } & \ldots & \ldots & \ldots & \ldots & \ldots\end{array}$ & 94,2 & 51,4 & 3,8 & 18,4 \\
\hline $\begin{array}{llllll}\text { Canarias } & \ldots & \ldots & \ldots & \ldots & \ldots \\
\end{array}$ & 81,7 & 49,8 & 2,8 & 9,4 \\
\hline 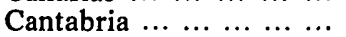 & 88,6 & 68,2 & 6,2 & 35,3 \\
\hline Castilla-León . ... . . . . . . . . & 85,8 & 52,4 & 4,1 & 12,8 \\
\hline Castilla-La Mancha... ... & 87,3 & 43,8 & 2,8 & 6,2 \\
\hline Cataluña $\ldots \ldots \ldots \ldots$ & 98,0 & 76,7 & 7,6 & 19,5 \\
\hline Comunidad Valenciana... & 96,8 & 66,0 & 4,8 & 13,6 \\
\hline Extremadura... $\ldots \ldots \ldots$ & 81,3 & 34,0 & 3,6 & 3,9 \\
\hline $\begin{array}{llllll}\text { Galicia } & \ldots & \ldots & \ldots & \ldots & \ldots\end{array}$ & 68,6 & 47,7 & 3,8 & 14,8 \\
\hline $\begin{array}{llllll} & & & & & \\
\text { Madrid } & \ldots & \ldots & \ldots & \ldots & \ldots\end{array}$ & 98,5 & 81,9 & 14,3 & 35,1 \\
\hline $\begin{array}{cccccc}\text { Murcia } & \ldots & \ldots & \ldots & \ldots & \ldots\end{array}$ & 95,2 & 58,7 & 2,9 & 8,6 \\
\hline $\begin{array}{llllll}\text { Navarra } & \ldots & \ldots & \ldots & \ldots & \ldots\end{array}$ & 96,8 & 77,3 & 10,0 & 24,9 \\
\hline $\begin{array}{lllll}\text { País Vasco } & \ldots & \ldots & \ldots & \ldots\end{array}$ & 95,7 & 81,2 & 9,3 & 44,4 \\
\hline 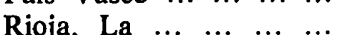 & 92,9 & 71,4 & 4,6 & 14,2 \\
\hline Ceuta y Melilla .......... & 92,5 & 59,7 & 2,3 & 8,3 \\
\hline
\end{tabular}


El uso del frigorifico se ha generalizado en los hogares españoles. Más del 90 por 100 de los hogares disponen de él. Madrid, Cataluña y Valencia, el País Vasco, Navarra, La Rioja, Aragón, Murcia y las Baleares rebasan el promedio nacional, y en las restantes Comunidades disponen también de él entre el 80 y el 90 por 100 de los hogares. En Galicia, con el valor mínimo, disponen de él más de dos terceras partes de sus hogares.

La lavadora automática es otro logro del hogar español. Dos terceras partes de los hogares españoles disponen de ella, aunque su uso es más desigual entre las Comunidades Autónomas, si bien se está generalizando con rapidez. En Madrid y el País Vasco disponen de ella cuatro de cada cinco hogares, y le siguen Cataluña, Navarra, La Rioja y Asturias, con más del 70 por 100 de sus hogares en uso de este aparato, y Aragón, Cantabria y Valencia, con dos terceras partes de sus hogares disponiendo de él. En las demás Comunidades su uso alcanza desde la tercera parte a la mitad de los hogares.

El lavavajillas es de uso todavía muy limitado. Madrid, Navarra, Cataluña y Aragón destacan, por encima del promedio nacional, en orden al uso de este aparato.

El uso del aspirador está más extendido que el del lavavajillas y alcanza a algo más de la sexta parte de los hogares españoles. El País Vasco, Madrid y Cantabria, Navarra, Baleares, Cataluña y Canarias son las Comunidades Autónomas en que está más extendido su uso.

\section{AParatos DE música E IMAGEN}

Los hogares españoles están equipados con los siguientes aparatos de música e imagen: 


\begin{tabular}{|c|c|c|c|c|c|}
\hline & Radio & $T V$ color & $\begin{array}{c}\text { Magne- } \\
\text { tofono } \\
\text { o radio- } \\
\text { cassette }\end{array}$ & Tocadiscos & $\begin{array}{c}\text { Cámara } \\
\text { fotográfica }\end{array}$ \\
\hline & & \multicolumn{3}{|c|}{ Porcentajes } & . \\
\hline EN CONJUNTO ... & 67,0 & 26,5 & 38,7 & 20,1 & 33,6 \\
\hline $\begin{array}{llll}\text { Andalucía } & \ldots & \ldots & \ldots\end{array}$ & 56,9 & 24,0 & 35,5 & 14,3 & 22,5 \\
\hline $\begin{array}{lllll}\text { Aragón } & \ldots & \ldots & \ldots & \ldots \\
\end{array}$ & 64,4 & 26,9 & 38,5 & 17,0 & 31,4 \\
\hline $\begin{array}{lllll}\text { Asturias... } & \ldots & \ldots & \ldots \\
\end{array}$ & 66,5 & 20,1 & 34,0 & 12,7 & 34,1 \\
\hline Baleares... . . . . . . . & 55,5 & 34,8 & 27,3 & 17,7 & 33,0 \\
\hline Canarias .................. & 72,1 & 29,7 & 30,5 & 16,0 & 22,7 \\
\hline $\begin{array}{llll} & & & \\
\text { Cantabria } & \ldots & \ldots & \ldots\end{array}$ & 78,4 & 18,2 & 38,1 & 20,5 & 37,8 \\
\hline Castilla-León $\quad \ldots \quad \ldots$ & 68,7 & 16,1 & 33,0 & 10,7 & 26,2 \\
\hline Castilla-La Mancha.. & 58,3 & 15,8 & 27,8 & 9,2 & 18,4 \\
\hline Cataluña... $\ldots \ldots \ldots$ & 82,2 & 35,8 & 46,8 & 32,7 & 43,9 \\
\hline \multicolumn{6}{|l|}{ Comunidad Valencia- } \\
\hline $\begin{array}{cccccc}\text { na } & \ldots & \ldots & \ldots & \ldots & \ldots\end{array}$ & 65,8 & 36,3 & 45,0 & 22,1 & 41,0 \\
\hline Extremadura... $\cdot \ldots \ldots$ & 56,9 & 14,2 & 29,5 & 7,4 & 15,6 \\
\hline $\begin{array}{lllll}\text { Galicia } & \ldots & \ldots & \ldots & \ldots\end{array}$ & 65,2 & 12,2 & 19,8 & 12,0 & 22,1 \\
\hline 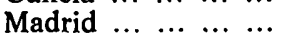 & 63,6 & 35,9 & 49,7 & 32,4 & 50,2 \\
\hline $\begin{array}{lllll}\text { Murcia } & \ldots & \ldots & \ldots & \ldots\end{array}$ & 49,6 & 24,3 & 36,6 & 13,6 & 24,1 \\
\hline 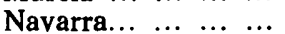 & 73,7 & 21,6 & 53,8 & 18,0 & 43,3 \\
\hline País Vasco ... ... ... & 79,8 & 16,7 & 45,0 & 21,2 & 41,6 \\
\hline Rioja, La $\ldots \ldots$... & 81,6 & 22,2 & 29,2 & 12,5 & 26,4 \\
\hline Ceuta y Melilla... ... & 50,0 & 72,1 & 50,9 & 25,7 & 38,7 \\
\hline
\end{tabular}

Las dos terceras partes de los hogares españoles disponen de aparato de radio. Su disponibilidad en las Comunidades Autónomas varía de la mitad de los hogares en Murcia y en Ceuta y Melilla, al 82 por 100 en Cataluña, que juntamente con La Rioja, País Vasco y Navarra, Cantabria y Canarias destacan sobre las demás y superan el promedio nacional.

El 72 por 100 de los hogares españoles disponen de televisión en blanco y negro, con una disponibilidad muy similar entre las Autonomías, entre el 70 y el 78 por 100 , sobresaliendo el País Vasco, con el 84 por 100 , con los mínimos del 59 por 100 en Baleares y del 34 por 100 en Ceuta y Melilla.

La televisión en color no está todavía tan extendida. Sólo la cuarta parte de los hogares disponen de ella. Por Comunidades destacan Valencia, Madrid, Cataluña, Baleares, Canarias y Aragón, y excepcionalmente, Ceuta y Melilla. Las demás Comunidades tienen televisor en color entre el 12 y el 24 por 100 de sus hogares.

Algo más de la tercera parte de los hogares españoles tienen magnetófono o radio-cassette. Sobresalen, en este aspecto, las Co- 
munidades Autónomas de Navarra y País Vasco, Madrid, Cataluña y Valencia, así como Ceuta y Melilla.

La quinta parte de los hogares españoles disponen de tocadiscos, sobresaliendo las Comunidades Autónomas de Madrid y Cataluña, Valencia, País Vasco, Cantabria y Ceuta y Melilla.

Finalmente, disponen de cámara fotográfica un tercio de los hogares españoles, si bien sobresalen por su mayor disponibilidad las Comunidades Autónomas de Madrid, Cataluña y Valencia, Navarra y el País Vasco, Asturias, Cantabria y Ceuta y Melilla.

\section{Vehículos}

Los hogares españoles disponen de los siguientes vehículos:

\begin{tabular}{|c|c|c|c|}
\hline & Autombviles & Motocicletas & Bicicletas \\
\hline & & orcentajes & \\
\hline EN conJunTo $\ldots \ldots \ldots \ldots$ & 51,5 & 12,8 & 7,5 \\
\hline $\begin{array}{lllllll}\text { Andalucía } & \ldots & \ldots & \ldots & \ldots & \ldots & \ldots\end{array}$ & 42,7 & 20,4 & 5,0 \\
\hline $\begin{array}{lllllllllllllll}\text { Aragon } & \ldots & \ldots & \ldots & \ldots & \ldots & \ldots & \ldots\end{array}$ & 52,0 & 12,6 & 18,7 \\
\hline $\begin{array}{lllllllll}\text { Asturias } & \ldots & \ldots & \ldots & \ldots & \ldots & \ldots & \ldots\end{array}$ & 47,8 & 3,9 & 3,5 \\
\hline $\begin{array}{cccccccc}\text { Baleares } & \ldots & \ldots & \ldots & \ldots & \ldots & \ldots & \ldots\end{array}$ & 61,1 & 24,5 & 14,5 \\
\hline 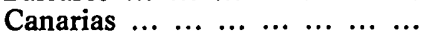 & 50,1 & 5,1 & 5,6 \\
\hline $\begin{array}{lllllll}\text { Cantabria } & \ldots & \ldots & \ldots & \ldots & \ldots & \ldots\end{array}$ & 53,0 & 13,3 & 10,1 \\
\hline $\begin{array}{llllll}\text { Castilla-Leon } & \ldots & \ldots & \ldots & \ldots & \ldots\end{array}$ & 44,7 & 7,8 & 7,9 \\
\hline Castilla-La Mancha ... ... ... ... & 40,7 & 17,0 & 10,2 \\
\hline 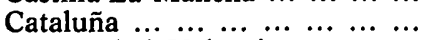 & 61,1 & 12,3 & 13,4 \\
\hline Comunidad Valenciana... ... ... & 60,6 & 20,0 & 9,3 \\
\hline 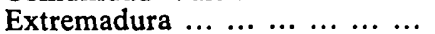 & 35,1 & 15,9 & 3,7 \\
\hline $\begin{array}{lllllllll}\text { Galicia } & \ldots & \ldots & \ldots & \ldots & \ldots & \ldots & \ldots\end{array}$ & 46,2 & 10,2 & 3,9 \\
\hline $\begin{array}{llllllll}\text { Madrid } & \ldots & \ldots & \ldots & \ldots & \ldots & \ldots & \ldots\end{array}$ & 54,2 & 3,3 & 1,2 \\
\hline $\begin{array}{llllllll}\text { Murcia } & \ldots & \ldots & \ldots & \ldots & \ldots & \ldots & \ldots\end{array}$ & 54,2 & 26,4 & 7,6 \\
\hline $\begin{array}{llllllll} & \text { Navarra } & \ldots & \ldots & \ldots & \ldots & \ldots & \ldots\end{array}$ & 64,8 & 14,8 & 6,7 \\
\hline 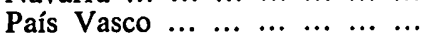 & 54,1 & 3,6 & 4,5 \\
\hline 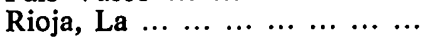 & 51,7 & 13,4 & 14,9 \\
\hline $\begin{array}{lllllll}\text { Ceuta } & \text { y Melilla } & \ldots & \ldots & \ldots & \ldots & \ldots\end{array}$ & 47,3 & 4,9 & 15,9 \\
\hline
\end{tabular}

Disponen de vehículos automóviles algo más de la mitad de los hogares españoles. Hasta diez Comunidades Autónomas destacan en este aspecto, por cuanto superan el promedio nacional: Navarra, Cataluña y Baleares, Valencia, Madrid, Murcia y País Vasco, Cantabria, Aragón y La Rioja. Las demás Comunidades ofrecen un 
mínimo de un tercio de hogares con automóvil —Extremadura-, y la mitad, como máximo - Canarias-.

Trece hogares de cada cien disponen de motocicleta. La dispersión de valores entre las distintas Comunidades Autónomas es muy acusada. Murcia, Baleares, Valencia y Andalucía figuran a la cabe$\mathrm{za}$, disponiendo la cuarta o quinta parte de los hogares este medio de locomoción. A segundo nivel, se sitúan, por encima del promedio nacional, Castilla-La Mancha, Extremadura, Navarra, La Rioja y Cantabria.

Sólo ocho hogares de cada cien disponen de bicicleta. Aragón, Ceuta y Melilla, La Rioja, Baleares, Cataluña, Castilla-La Mancha y Cantabria se sitúan por encima del promedio nacional. 
REVL-1984, núm. 221. BALLESTER ROS, IGNACIO. EL EQUIPAMIENTO Y OTRAS CARACTERIST...

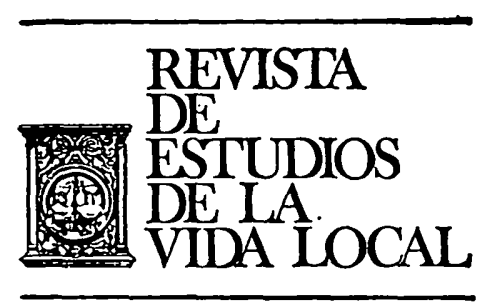

IV. JURISPRUDENCIA 
REVL-1984, núm. 221. BALLESTER ROS, IGNACIO. EL EQUIPAMIENTO Y OTRAS CARACTERIST...

REVL-1984, núm. 221. BALLESTER ROS, IGNACIO. EL EQUIPAMIENTO Y OTRAS CARACTERIST... 